\section{LA-UR- $10-0 / 101$}

Approved for public release;

distribution is unlimited.

Title:

Author(s):

Intended for:

APS Topical Conference on Shock Compression, June 28 July 4, 2009 Nashville, TN
High Velocity Properties of the Dynamic Frictional Force Between Ductile Metals

J.E. Hammerberg, B.L. Holian, T.C. Germann, R. Ravelo

Los Alamos National Laboratory, an affirmative action/equal opportunity employer, is operated by the Los Alamos National Security, LLC for the National Nuclear Security Administration of the U.S. Department of Energy under contract DE-AC52-06NA25396. By acceptance of this article, the publisher recognizes that the U.S. Government retains a nonexclusive, royalty-free license to publish or reproduce the published form of this contribution, or to allow others to do so, for U.S. Government purposes. Los Alamos National Laboratory requests that the publisher identify this article as work performed under the auspices of the U.S. Department of Energy. Los Alamos National Laboratory strongly supports academic freedom and a researcher's right to publish; as an institution, however, the Laboratory does not endorse the viewpoint of a publication or guarantee its technical correctness. 


\title{
HIGH VELOCITY PROPERTIES OF THE DYNAMIC FRICTIONAL FORCE BETWEEN DUCTILE METALS
}

\author{
J. E. Hammerberg', B. L. Holian', T. C. Germann', and R. Ravelo ${ }^{2}$ \\ 'Los Alamos National Laboratory, Los Alamos NM 87545 \\ ${ }^{2}$ Physics Dept., University of Texas, El Paso, El Paso TX 79968
}

\begin{abstract}
The high velocity properties of the tangential frictional force between ductile metal interfaces seen in large-scale NonEquilibrium Molecular Dynamics (NEMD) simulations are characterized by interesting scaling behavior. In many cases a power law decrease in the frictional force with increasing velocity is observed at high velocities. We discuss the velocity dependence of the high velocity branch of the tangential force in terms of structural transformation and ultimate transition, at the highest velocities, to confined fluid behavior characterized by a critical strain rate. The particular case of an $\mathrm{Al} / \mathrm{Al}$ interface is discussed.
\end{abstract}

Keywords: Molecular dynamics, frictional force, plastic deformation, sliding friction, aluminum.

PACS: $62.20 .-\mathrm{x}, 62.20 . \mathrm{Qp}, 62.50 .-\mathrm{p}, 61.66 . \mathrm{Bi}, 68.15 .+\mathrm{e}, 68.35$.Af.

\section{INTRODUCTION}

Velocity weakening of the tangential frictional force between ductile metals at high sliding velocities has been observed both experimentally [1-3] and in large scale NEMD simulations for a variety of material pairs including Lennard-Jones/LennardJones, $\mathrm{Cu} / \mathrm{Cu}, \mathrm{Cu} / \mathrm{Ag}, \mathrm{Ta} / \mathrm{Al}$ and $\mathrm{Al} / \mathrm{Al}[4-8]$. The decrease of the tangential force, $F_{t}$, with increasing velocity for NEMD configurations with fixed normal load, tangential velocity, and temperature imposed in a small boundary region located at $\pm \frac{L}{2}$, where $L$ is the thickness normal to the sliding direction, is very well represented by a power law dependence, $F_{1} \sim\left(\frac{v}{v_{c}}\right)^{-\beta}$, when the relative sliding velocity, $v$, exceeds a critical velocity, $v_{c}$. Typically, $v_{c}$ is of order $100 \mathrm{~m} / \mathrm{s}$ in the NEMD simulations and the power law behavior persists to the highest velocities studied, of order $3 \mathrm{~km} / \mathrm{s}$ with $\beta \cong \frac{3}{4}$. The origin of this weakening may be found in the instability of the sliding interface to structural transformation induced by large plastic deformation resulting, at the highest velocities, in the formation of a third-body confined fluid layer. We discuss some of the properties of the high velocity regime for $\mathrm{Al}(111) / \mathrm{Al}(001)$ sliding in the following sections.

\section{METHOD}

Simulations for 1.4 million atom samples have been described in [9] for a range of temperatures and a normal load of $15 \mathrm{GPa}$ for an $\mathrm{Al}(111) / \mathrm{Al}(001)$ interface. Above a critical velocity, $v_{c}$, given by

$$
\mathrm{v}_{\mathrm{c}}=4 \frac{\bar{\kappa} \mathrm{T}^{*}}{\mathrm{f}_{\mathrm{c}} \mathrm{L}}\left(1-\frac{\mathrm{T}_{0}}{\mathrm{~T}^{*}}\right),
$$


the results for $F_{t}$ for different boundary temperatures could be rescaled onto a single curve

$$
\frac{F_{t}}{A} \equiv f=f_{c}\left(\frac{v}{v_{c}}\right)^{-\beta}
$$

Here $f_{c}$ is a critical force per unit area, and $A$ is the surface area. $T_{0}$ is the boundary temperature at $y= \pm \frac{L}{2}, \bar{\kappa}$ is the average thermal conductivity between $T_{0}$ and a critical interface temperature, $T^{*}$, and $\beta \cong \frac{3}{4}$. The calculated melting temperature for the Al EAM potentials used in these simulations [7] is $T_{m}=1130 \mathrm{~K}$ for $\mathrm{P}=15 \mathrm{GPa}$. Within the accuracy of these simulations, $\mathrm{T}^{*} \cong \mathrm{T}_{\mathrm{m}}$. Of particular interest is the interfacial temperature as a function of relative sliding velocity. Fig. 1 shows the interfacial temperature for three boundary temperatures, $\mathrm{T}_{0}=232,464$ and $696 \mathrm{~K}$.

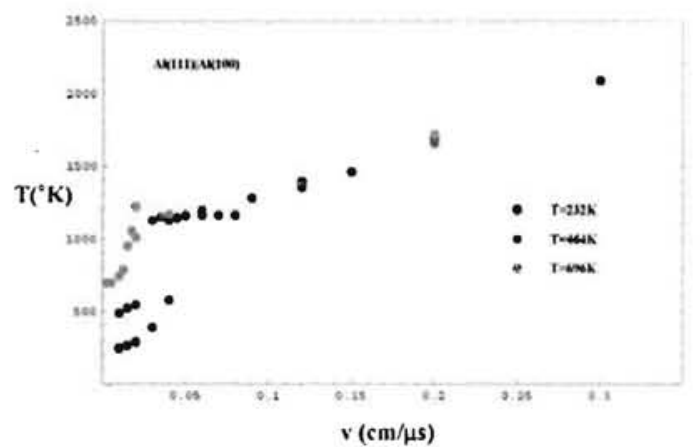

Figure 1. Interfacial temperature vs. velocity for boundary temperatures $T_{0}=232,464$ and $696 \mathrm{~K}$.

Whereas the tangential force obeys the scaling equation (2), the interfacial temperature at high velocities is a function of $v$ rather than $v / v_{c}$. Also, for velocities $v_{c} \leq v \leq v_{c}^{*}$, the interfacial temperature is constant and equal to $\mathrm{T}^{*}$.

\section{RESULTS}

For $\mathrm{v}_{\mathrm{c}} \leq \mathrm{v} \leq \mathrm{v}_{\mathrm{c}}^{*}$, the sliding interface becomes unstable to a structural transformation with a constant temperature transformation front at temperature $\mathrm{T}^{*}$ moving normal to the sliding direction transforming (111) material into (001) material. Figure 2 shows the steady state position of the sliding interface for $\mathrm{T}_{0}=232 \mathrm{~K}$.

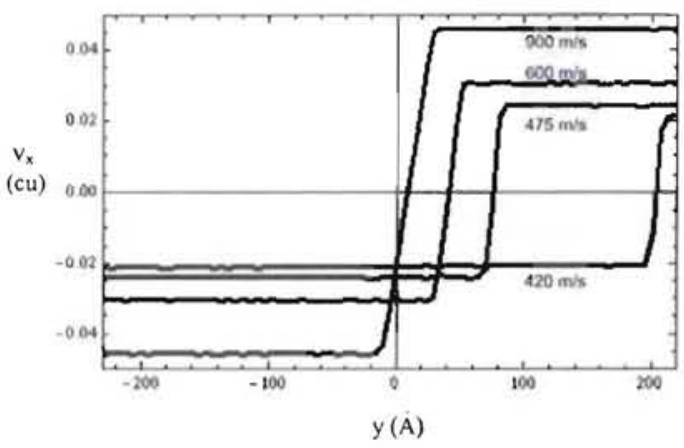

Figure 2. Tangential velocity, $v_{x}$, vs. normal distance, $y$, for boundary temperature $T_{0}=232 \mathrm{~K} . \mathrm{v}_{\mathrm{x}}$ in computational units, cu, with $1 \mathrm{cu}=9.823 \mathrm{~km} / \mathrm{s}$ and $\mathrm{y}$ in $\dot{A}$. Curves for relative velocities $420,475,600$, and $900 \mathrm{~m} / \mathrm{s}$ are shown and $\mathrm{v}_{\mathrm{c}} \leq \mathrm{v} \leq \mathrm{v}_{\mathrm{c}}^{*}, \mathrm{v}_{\mathrm{c}} \cong 420 \mathrm{~m} / \mathrm{s}$.

For $\mathrm{v} \cong \mathrm{v}_{\mathrm{c}} \cong 420 \mathrm{~m} / \mathrm{s}$, the front moves to nearly the boundary before the large thermal gradient stabilizes its position. As $\mathrm{v}$ increases, the position of the sliding interface approaches the initial sliding interface position which is attained for $v=v_{c}^{*}$ with $\frac{v_{c}^{*}}{v_{c}} \cong 2$. As $v$ approaches $v_{c}^{*}$, the interfacial velocity profile broadens as is evident in Fig. 2. For $v \geq v_{\mathrm{c}}^{*}$, a classical Couette flow pattern

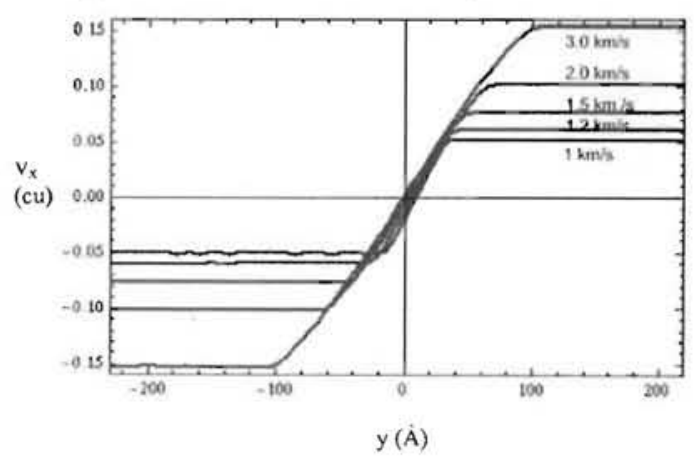

Figure 3. Tangential velocity, $v_{x}$, vs. normal distance, $y$, for boundary temperature $T_{0}=232 \mathrm{~K} . \mathrm{v}_{\mathrm{x}}$ in computational units, cu, with $1 \mathrm{cu}=9.823 \mathrm{~km} / \mathrm{s}$ and $\mathrm{y}$ in $\hat{\mathrm{A}}$. Curves for relative velocities $1.0,1.2,1.5,2.0$, and $3.0 \mathrm{~km} / \mathrm{s}$ are shown and $v \geq v_{\mathrm{c}}^{*}$. 
develops for the average tangential velocity as a function of distance normal to the interface. This is shown in detail in Fig. 3 where the tangential velocity, $v_{x}$, is plotted as a function of normal distance, $\mathrm{y}$, for relative velocities 1.0, 1.2, 1.5, 2.0, and $3.0 \mathrm{~km} / \mathrm{s}$ and a boundary temperature of $\mathrm{T}_{0}=$ $232 \mathrm{~K}$.

The slope of the velocity profile is nearly independent of the sliding velocity with a critical strain rate $\dot{\varepsilon}_{\mathrm{c}}=\frac{1}{2} \frac{\partial v_{x}}{\partial y}=0.75 \times 10^{11} \mathrm{~s}^{-1}$. Fig. 4 shows the dependence of the critical strain rate on the boundary temperature. The tangential velocity profiles, $v_{x}$ vs. normal distance $y$, are shown for a constant relative velocity of $2 \mathrm{~km} / \mathrm{s}$ and boundary temperatures 232,464 and $696 \mathrm{~K}$.

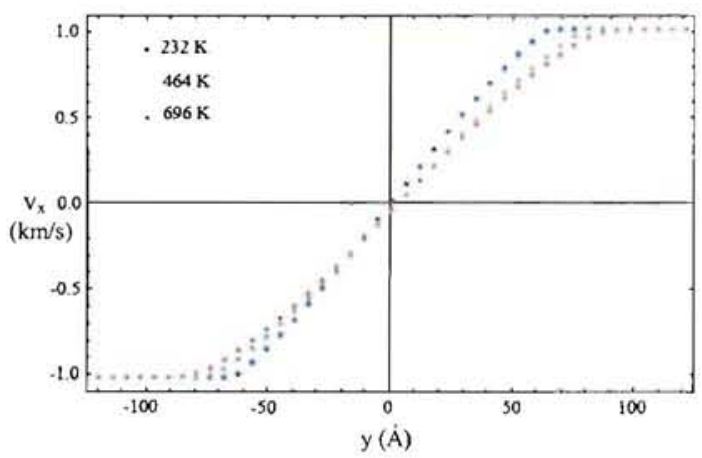

Figure 4. Tangential velocity, $v_{x}$, vs. normal distance, $y$, for boundary temperatures $T_{0}=232$ (upper), 464 (middle) and 696 (lower) K. Relative velocity $=2.0 \mathrm{~km} / \mathrm{s}$.

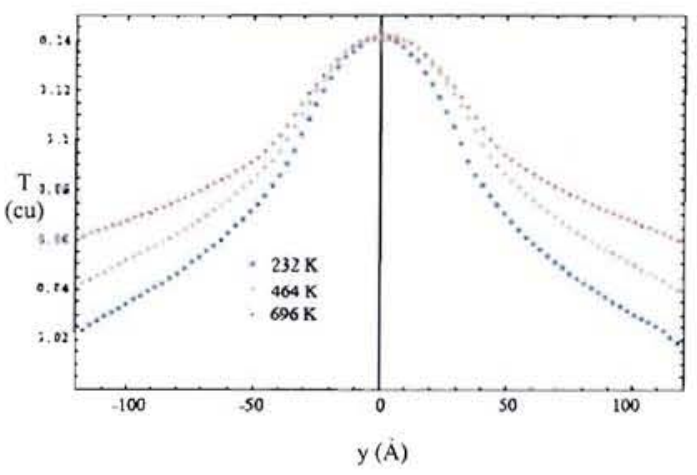

Figure 5. Temperature vs. normal distance, y, for boundary temperatures $T_{0}=232$ (lower), 464 (middle) and 696 (upper) $\mathrm{K}$. Temperature in cu with $1 \mathrm{cu}=11605$ $\mathrm{K}$. Relative velocity $=2.0 \mathrm{~km} / \mathrm{s}$.
Fig. 5 shows the temperature profiles averaged over the transverse directions for the same conditions. The quadratic Couette temperature dependence in the fluid region is evident. In this figure one computational unit of temperature (cu) is $11605 \mathrm{~K}$.

\section{DISCUSSION}

The velocity dependence of constitutive material quantities in the high velocity regime can be analyzed in terms of energy flow and thermal diffusion in the nonequilibrium steady state. Denoting by $T_{m}$ the melting temperature at the Couette boundary, $T(0)$ the interfacial temperature, and $\bar{\kappa}_{f}$ the temperature averaged thermal conductivity in the fluid region, consideration of a control volume parallel to the sliding interface results in the following expression for the interfacial temperature for $v \geq v_{\mathrm{c}}^{*}$.

$$
\mathrm{T}(0)=\mathrm{T}_{\mathrm{m}}+\frac{1}{8}\left[\frac{\mathrm{f}}{\overline{\mathrm{K}}_{\mathrm{f}} \dot{\varepsilon}_{\mathrm{c}}}\right] \mathrm{v}^{2} .
$$

It is also possible to express $T(0)$ in terms of fluid dynamical quantities:

$$
\begin{aligned}
& \mathrm{T}(0)=\mathrm{T}_{\mathrm{m}}+\frac{1}{8}\left(\overline{\frac{\kappa_{\mathrm{f}}}{\eta}}\right)^{-1} \mathrm{v}^{2}, \\
& \mathrm{~T}(0)=\mathrm{T}_{\mathrm{m}}+\frac{1}{8}\left(\overline{\frac{\mathrm{c}_{\mathrm{v}}}{\mathrm{Pr}}}\right)^{-1} \mathrm{v}^{2},
\end{aligned}
$$

where $\dot{\varepsilon}_{c}$ is the critical strain rate in the Couette regime, $\eta$ is the fluid viscosity, $c_{v}$ the specific heat, $\mathrm{Pr}$ is the Prandt'l number, and the averages are temperature averages between $T(0)$ and $T_{m}$. Similarly, the position dependence of the temperature in the fluid region is given by

$$
T(y)=T(0)-\frac{1}{2}\left[\frac{f \dot{\varepsilon}_{c}}{\left\langle\kappa_{f}\right\rangle}\right] y^{2},
$$


or

$$
T(y)=T(0)-\frac{1}{2}\left\langle\frac{\kappa_{f}}{\eta}\right\rangle^{-1} \dot{\varepsilon}_{c}^{2} y^{2}
$$

where the brackets denote a spatial average between the interface and $y$.

These relations and the scaled form for $f$ imply relationships for $T(0)$ as a function of velocity and the dependence of the critical strain rate on $v_{c}$. From equations (2) and (3) we have

$$
T(0)=T_{m}+\frac{1}{8}\left[\frac{f_{c} v_{c}^{\beta}}{\bar{\kappa}_{f} \dot{\varepsilon}_{c}}\right] v^{1+\alpha},
$$

where $\alpha=1-\beta \cong \frac{1}{4}$, giving a weakly supra-linear increase with velocity which is consistent with Fig. 1. Alternatively, from equation (4), the increase with velocity of the interfacial temperature is related to the temperature dependence of $\bar{\kappa}_{\mathrm{r}}$ and temperature dependence of the confined fluid viscosity. From the simulation result that the interfacial temperature is independent of the boundary temperature in the steady state, it also follows that

$$
\dot{\varepsilon}_{\mathrm{c}}=\mathrm{B} \frac{\mathrm{f}_{\mathrm{c}}}{\overline{\mathrm{\kappa}}_{\mathrm{r}}} \mathrm{v}_{\mathrm{c}}^{\beta},
$$

where $\mathrm{B}$ is a constant. These expressions also relate $\mathrm{f}_{\mathrm{c}}, \dot{\varepsilon}_{\mathrm{c}}$ and $\eta\left(\mathrm{T}_{\mathrm{m}}\right)$.

\section{CONCLUSIONS}

We have discussed the high velocity behavior of the tangential frictional force between ductile metals as seen in large scale NEMD simulations taking an $\mathrm{Al}(111) / \mathrm{Al}(001)$ incommensurate interface at $15 \mathrm{GPa}$ as an example. The results of these simulations may be expressed in a scaled form in which the velocity dependence for the tangential force is a function of $v / v_{c}$ where $v_{c}$ is a temperature dependent critical velocity. For velocities above $v_{c}$ but below a second critical velocity, $v_{c}^{*}$, the interface is unstable relative to structural transformation which in the case of $\mathrm{Al}(111) / \mathrm{Al}(001)$ manifests itself as a moving transformation front which coin- cides with the sliding interface and whose temperature is near the bulk melting temperature. Above $v_{c}^{*}$, a confined fluid region is stabilized at the interface with a Couette flow pattern in velocity with properties that may be explained in terms of microscopic fluid parameters and the scaled frictional force, and which exhibits a velocity independent critical strain rate for fixed boundary temperature.

\section{ACKNOWLEDGEMENTS}

This work was performed under the auspices of the U.S. Dept. of Energy under contract DE-AC5206NA25396.

\section{REFERENCES}

1. Bowden, F. P. and Freitag, E. H., "The Friction of Solids at Very High Speeds", Proc. Roy. Soc. (Lond.) Ser. A.,1958, Vol. 248, pp. 350-367.

2. Rousculp, C. A. et al., "Dynamic Friction Experiments at the ATLAS Pulsed Power Facility", Proc. 2006 Int. Conf. on MegaGauss Magnetic Field Generation and Related Topics, 2007.

3. Winter, R. E., et al., "Mechanisms of ShockInduced Dynamic Friction”, J. Phys. D., 2006, Vol. 39 , pp. 5043-5053.

4. Hammerberg, J.E. et al., "Modeling High Speed Friction at Ductile Metal Interfaces", LANL Report, LA-UR-02-4654, 2002.

5. Hammerberg, J.E. et al., "Nonlinear Dynamics and the Problem of Slip at Material Interfaces", Physica, 1999, D123, pp. 330-340.

6. Mikulla, R.P. et al., "Dislocation Nucleation and Dynamics at Sliding Interfaces”, Mater. Res. Soc. Symp. Proc., 1998, 522, 385-391.

7. Hammerberg, J.E. et al., "Nonequilibrium Molecular Dynamics Simulations of Metallic Friction at $\mathrm{Ta} / \mathrm{Al}$ and $\mathrm{Cu} / \mathrm{Ag}$ Interfaces", Met. and Mat. Trans. A, 2004, 34A, pp. 2741-2745.

8. Hammerberg, J. E., et al., Shock Compression of Condensed Matter, 2005 (M.D. Furnish, M. Elert, Russell, T.P., and C.T. White, eds.), part I, pp. 391394.

9. Hammerberg, J. E., et al., Shock Compression of Condensed Matter, 2007 (M. Elert, M.D. Furnish, R. Chau, N. Holmes, and J. Nguyen, eds.), pp. 309312. 